\title{
Qualidade de sementes de tomate influenciada pelos tratamentos térmi- co e osmótico
}

\author{
Flávio de Souza Lopes; Cláudia Antonia Vieira Rossetto \\ UFRRJ, Depto. Fitotecnia, C. Postal 74511, 23890-000 Seropédica-RJ; E-mail: cavrosse@ufrrj.br
}

\begin{abstract}
RESUMO
O tratamento térmico via calor seco, visando o controle de patógenos associado às sementes, pode causar danos à qualidade fisiológica das mesmas, que podem ser recuperados, por meio do condicionamento osmótico. Avaliou-se o efeito do tratamento térmico via calor seco na qualidade sanitária e fisiológica das sementes de tomate, assim como a influência do condicionamento osmótico na qualidade fisiológica das sementes submetidas à termoterapia. As sementes das cultivares Quadrado Mix, Topmec e Calmec VFN foram expostas ao tratamento térmico a $70 ; 72,5$ e $75^{\circ} \mathrm{C}$, em estufa com circulação forçada, por períodos de zero, 48; 96 e 120 horas. O período zero, sem a aplicação de calor, foi considerado como testemunha. Posteriormente, estas sementes foram avaliadas quanto à sanidade, condicionadas em solução aerada de polietilenoglicol a 0,0 e -1,0 MPa e submetidas à avaliação do teor de água, da germinação e do vigor. Pelos resultados pode-se concluir que o tratamento térmico via calor seco não foi eficiente em eliminar os patógenos associados às sementes de tomate, dependendo da cultivar avaliada. Períodos prolongados de exposição ao calor seco causaram danos à qualidade fisiológica das sementes de tomate, que não foram recuperados com o subseqüente condicionamento osmótico.
\end{abstract}

Palavras-chave: Lycopersicum esculentum, termoterapia, sanidade, germinação.

\begin{abstract}
Tomato seeds quality affected by dry heat and osmotic treatments

The dry heat treatment aiming to control microorganisms in the seeds can cause damage to the physiological quality of seeds. Osmotic conditioning can be useful tool for recovering the physiological quality of the heat treated seeds. The study was carried out to evaluate the effects of dry heat treatments on the tomato seeds sanitary and physiologic quality, as well as to analyze the effect of the osmotic conditioning on seeds submitted to dry heat treatment. The seeds of cultivars Quadrado Mix, Topmec and Calmec VFN were heated at $70 ; 72.5$ and $75^{\circ} \mathrm{C}$ by periods of zero, 48; 96 and 120 hours. After the heat treatment, the seeds were evaluated for the presence of pathogens and were submitted to the osmotic conditioning using polyethylenoglicol in the 0.0 and -1.0 MPa osmotic potentials. Seed moisture content, germination test and vigor tests were performed. The dry heat treatment did not eliminate the microorganisms in the tomato seeds, depending on variety evaluted. The prolonged periods of dry heat treatments caused severe seed damage and the osmotic conditioning treatment was not able to improve the seed quality.
\end{abstract}

Keywords: Lycopersicum esculentum, thermoterapy, seed health, germination.

\section{(Recebido para publicação em 14 de agosto de 2003 e aceito em 7 de junho de 2004)}

$\mathrm{P}$ atógenos como Fusarium oxysporum f.sp. lycopersici, Fusarium oxysporum f.sp. redolens, Cladosporium spp., Aspergillus spp. e Penicillium spp. podem estar associados às sementes de tomate (Lycopersicon esculentum Mill) (Muniz, 2001). No entanto, com o intuito de controlar ou de erradicar estes patógenos, vários métodos alternativos têm sido propostos em substituição ao uso de produtos químicos, entre eles, o tratamento térmico via calor seco (Menten, 1995, Perleberg e Sperandio, 1998, Trigo et al., 1998, Machado, 2000; Martins et al., 2001).

A exposição ao calor seco a $70^{\circ} \mathrm{C}$ por 96 horas controlou Xanthomonas campestris pv. vesicatoria, bem como, Aspergillus spp. e Rhizopus spp., sem comprometer a germinação das sementes de tomate, apesar de provocar redução do vigor e alterações na estrutura destas, como na região dos tricomas
(Silva et al., 2002). Também Muniz (2001), trabalhando com a mesma espécie, verificou que a exposição ao calor seco a $70^{\circ} \mathrm{C}$, por períodos de até 15 dias, controlou totalmente os microrganismos (Alternaria alternata, Fusarium spp. e Cladosporium fulvum), sem causar danos à germinação e ao vigor das sementes. Porém, como o tratamento térmico implica na elevação da temperatura, o teor de água das sementes pode interferir no nível de controle dos fungos e, principalmente na qualidade fisiológica das mesmas (Trigo et al., 1998).

Têm sido constatadas várias técnicas que vêm sendo aplicadas às sementes com o intuito de favorecer o desempenho destas, tais como, o condicionamento osmótico, que emprega solução de polietilenoglicol, de modo a promover a hidratação lenta das sementes (Hardegree e Emmerich, 1994). Com o emprego deste tratamento, tem sido ob- servado aumento da emissão da raiz primária (Mauromicale e Cavallaro, 1995), da velocidade de geminação (Frett et al., 1991), da emergência das plântulas (Muhyaddin e Wiebe, 1989) e do potencial de armazenamento das sementes (Rossetto et al., 2001 e Rossetto et al., 2002). Em sementes de tomate, as respostas a esta técnica podem ser diferentes em função da qualidade fisiológica inicialmente apresentada pelas sementes (Penãloza e Eira, 1993) e da cultivar avaliada (Mauromicale e Cavallaro, 1995). Para Penãloza e Eira (1993), o condicionamento osmótico, independente da cultivar, favoreceu a germinação apenas das sementes dos lotes de baixa qualidade fisiológica, pois estas precisam de maior período para promover a reorganização de suas membranas celulares, preparando-as para iniciar a germinação. Além disso, resultados favoráveis desta técnica não foram cons- 
tatados a $10^{\circ} \mathrm{C}$, porém apenas sob temperaturas de 20 e $30^{\circ} \mathrm{C}$ (Alvarado et al.,1987, Pill et al., 1991), condição que não foi considerada como de estresse, pois de acordo com Cavallaro et al. (1994), embora a germinação ocorra sob temperatura de $10 \mathrm{a} 35^{\circ} \mathrm{C}$, a temperatura ótima é de 20 a $25^{\circ} \mathrm{C}$ e sob temperatura abaixo de 12 a $15^{\circ} \mathrm{C}$, a germinação é reduzida.

Tendo em vista que as respostas do condicionamento osmótico têm sido distintas em função da cultivar e da qualidade fisiológica das sementes, que pode estar comprometida pelo tratamento térmico aplicado previamente às mesmas, este trabalho teve como objetivo avaliar o efeito do tratamento térmico via calor seco na qualidade sanitária e fisiológica das sementes de tomate, assim como analisar a influência do condicionamento osmótico na qualidade fisiológica das sementes submetidas a essa termoterapia.

\section{MATERIAL E MÉTODOS}

O estudo foi conduzido em laboratório da UFRRJ, em 2001. Foram utilizadas sementes comerciais de tomate (Lycopersicon esculentum Mill) das cultivares Quadrado Mix, Topmec e Calmec VFN, que permaneceram armazenadas em sacos de papel, em câmara fria $\left(18 \pm 2^{\circ} \mathrm{C}, 52 \pm 5 \%\right.$ de UR do ar), por dois meses, até o início dos experimentos.

O delineamento experimental adotado foi o inteiramente casualizado, em esquema fatorial $3 \times 3 \times 4 \times 2$ (três cultivares $\mathrm{x}$ três temperaturas da estufa $\mathrm{x}$ quatro períodos de exposição das sementes ao calor x duas concentrações de solução de polietilenoglicol), com quatro repetições.

Amostras de 5,0g das sementes das cultivares Quadrado Mix, Topmec e Calmec VFN foram acondicionadas em sacos de papel e submetidas ao tratamento térmico a $70 ; 72,5$ e $75^{\circ} \mathrm{C}$, em estufa com circulação de ar, por períodos de zero, 48; 96 e 120. O período zero, sem a aplicação do calor, foi considerado como testemunha. Após os períodos, as sementes foram submetidas ao teste de sanidade, pelo método do papel de filtro, conforme Brasil (1992),

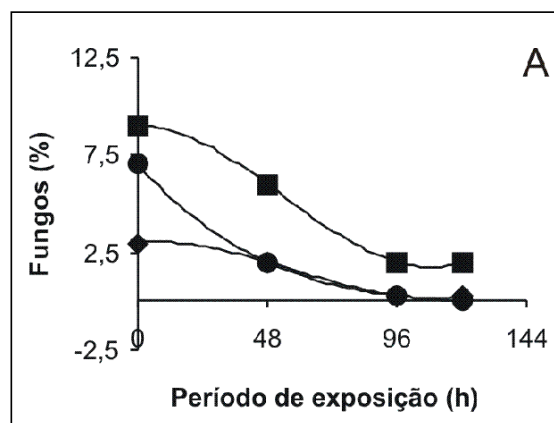

$(--) y=5 E-06 x^{3}-0,0009 x^{2}+0,0112 x+3,0 R^{2}=1,0000^{* *}$

(-) $y=-4 E-05^{3}+0,0011 x^{2}-0,0944 x+8,0 R^{2}=0,9999^{* *}$

(-) $y=4 \mathrm{E}-06 \mathrm{x}^{3}+0,00067 \mathrm{x}^{2}-0,0208 \mathrm{x}+7,5 \mathrm{R}^{2}=0,9999^{* *}$

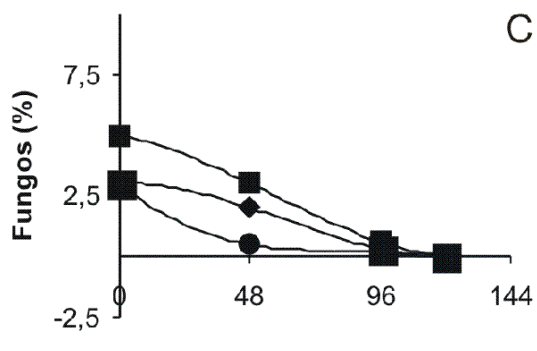

Período de exposição (h)

$(-\downarrow-) y=4 \mathrm{E}-06 \mathrm{x}^{3}-0,0007 \mathrm{x}^{2}+0,0833 \mathrm{x}+3,7 \mathrm{R}^{2}=0,9999 * *$

(-) $y=7 E-06 x^{3}-0,0010 x^{2}-0,1309 x+5,0 R^{2}=0,9999^{* *}$

(-) $y=7 \mathrm{E}-05 \mathrm{x}^{3}-0,0001 \mathrm{x}^{2}+0,0020 \mathrm{x}+7,0 \mathrm{R}^{2}=1,0000^{* *}$

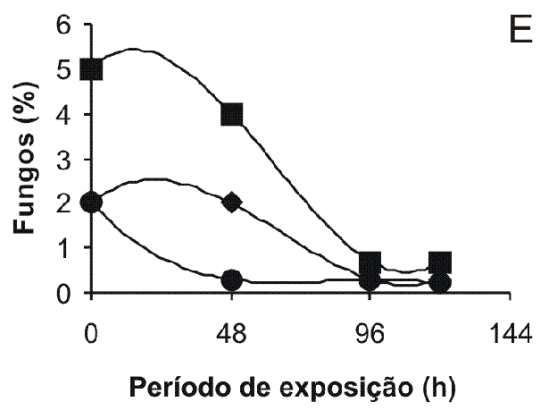

\section{E}

$(-\downarrow-) y=7 E-06 x^{3}-0,0014 x^{2}+0,0508 x+2 R^{2}=1,0000^{\star *}$

(- $y=1 E-05 x^{3}-0,0022 x^{2}+0,0590 x+5 R^{2}=1,0000^{* *}$

(-) $y=-4 E-06 x^{3}+0,0009 x^{2}-0,0695 x+2 R^{2}=1,0000^{\star *}$

$70^{\circ} \mathrm{C}(-\downarrow-), 72,5^{\circ} \mathrm{C}($

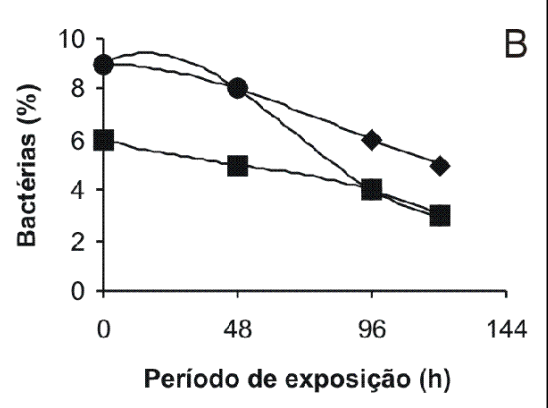

$(-\bullet) y=2 E-06 x^{3}-0,0005 x^{2}-0,0021 x+9 R^{2}=0.9999^{\star *}$

(-) $y=-2 E-06 x^{3}+0,0003 x^{2}-0,0319 x+6 R^{2}=1,0000^{\star *}$

(-) $y=1 E-050 x^{3}-0,0021 x^{2}+0,0576 x+9 R^{2}=0.9999^{* *}$

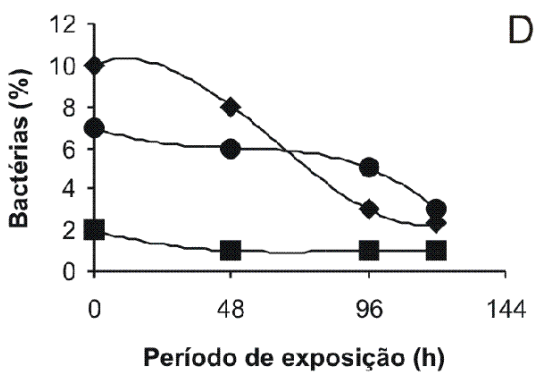

$(--) y=-2 E-06 x^{3}+0,0005 x^{2}-0,0396 x+10 R^{2}=1,0000 * *$

(-) $y=-2 E-06 x^{3}+0,0005 x^{2}-0,0396 x+2 R^{2}=1,0000 * *$

(-) $y=-7 \mathrm{E}-06 \mathrm{x}^{3}+0,0010 \mathrm{x}^{2}-0,0542 \mathrm{x}+7 \mathrm{R}^{2}=1,0000^{* * *}$

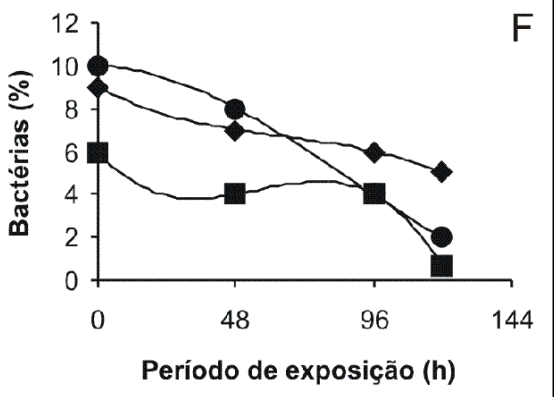

$(-\bullet) y=-4 E-06 x^{3}-0,0008 x^{2}-0,0715 x+9 R^{2}=1.0000^{\star *}$

(- $y=-2 E-05 x^{3}+0,0032 x^{2}-0,1525 x+6 R^{2}=1,0000^{* *}$

$(--) \mathrm{y}=4 \mathrm{E}-06 \mathrm{x}^{3+\cdot} 0,001 \mathrm{x}^{2}-0,0042 \mathrm{x}+10 \mathrm{R}^{2}=1,0000^{* *}$

Figura 1. Porcentagem de fungos e bactérias associados às sementes de tomate das cultivares Quadrado Mix (a), Topmec (b) e Calmec VFN (c), submetidas ao tratamento térmico. Rio de Janeiro, UFRRJ, 2001.

a $27 \pm 2^{\circ} \mathrm{C}$, em regime alternado de 12 horas de luz, por sete dias (Silva et al., 2002). Posteriormente, amostras de 1,5 g de sementes, imersas em $200 \mathrm{ml}$ de solução de polietilenoglicol a 0,0 e $-1,0$ $\mathrm{MPa}$, com base em Michel e Kaufmann (1973), por sete dias a $20^{\circ} \mathrm{C}$ na ausência de luz e secas sobre papel de filtro em condições de laboratório sem controle por 20 horas. Avaliaram-se o teor de água e da qualidade fisiológica (ger- minação e vigor, pelos testes de primeira contagem e condutividade elétrica) das sementes.

A determinação do teor de água foi realizada em quatro sub-amostras de 50 sementes, que foram submetidas ao método da estufa a $105 \pm 3^{\circ} \mathrm{C}$, durante 24 horas (Brasil, 1992). O teste de germinação foi realizado em quatro sub-amostras de 50 sementes, que foram mantidas em 8 horas de luz a $30^{\circ} \mathrm{C}$ e por dezesseis 

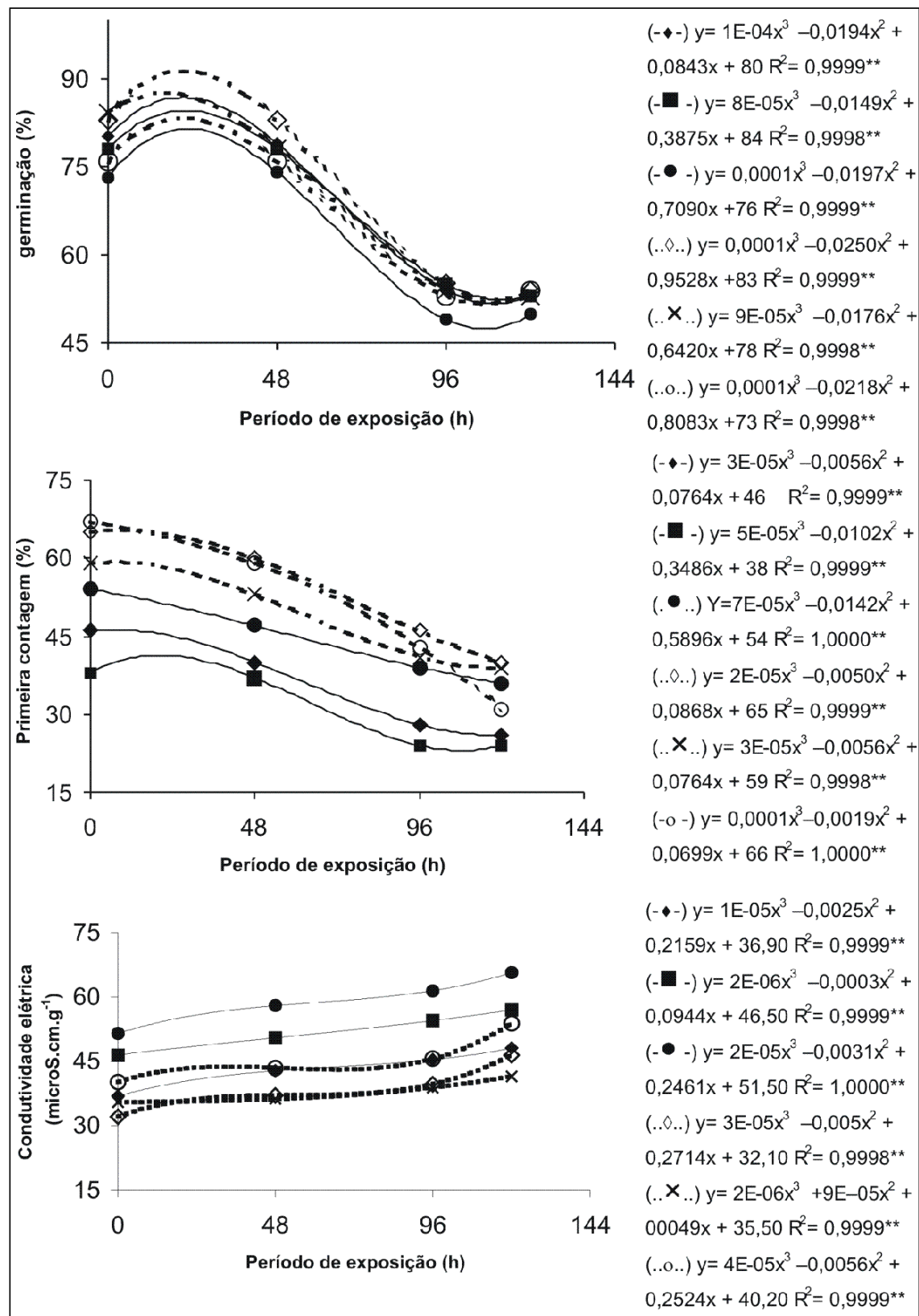

$70^{\circ} \mathrm{C}(--), 72,5^{\circ} \mathrm{C}(-\mathbf{m}-)$ e $75^{\circ} \mathrm{C}(-\bullet-)$ - sem condicionamerto osmótico

$70^{\circ} \mathrm{C}(. \diamond .), 72,.5^{\circ} \mathrm{C}(. . \times$.. $)$ e $75^{\circ} \mathrm{C}(. .0 .$.$) - com condicionamento osmótico$

Figura 2. Porcentagem de germinação e de plântulas normais na primeira contagem e condutividade elétrica, obtidos de sementes de tomate de três cultivares, submetidas ao tratamento térmico e ao condicionamento osmótico. Rio de Janeiro, UFRRJ, 2001.

horas de escuro a $20^{\circ} \mathrm{C}$, e avaliadas aos cinco e quatorze dias (Brasil, 1992). O teste de primeira contagem da germinação foi realizado em conjunto com o teste anterior, visando obter a porcentagem de plântulas normais aos cinco dias da instalação do teste. O teste de condutividade elétrica foi realizado em quatro sub-amostras de 50 sementes, que foram pesadas e colocadas em copos plásticos, contendo $50 \mathrm{ml}$ de água des-

$(-\downarrow) y=1 E-04 x^{3}-0,0194 x^{2}+$ $0,0843 x+80 R^{2}=0,9999 * *$

(-D -) $\mathrm{y}=8 \mathrm{E}-05 \mathrm{x}^{3}-0,0149 \mathrm{x}^{2}+$ $0,3875 x+84 R^{2}=0,9998^{* *}$

$(-) \mathrm{y}=0,0001 \mathrm{x}^{3}-0,0197 \mathrm{x}^{2}+$ $0,7090 x+76 R^{2}=0,9999^{* *}$

(..৬..) $y=0,0001 x^{3}-0,0250 x^{2}+$ $0,9528 x+83 R^{2}=0,9999^{* *}$

(.. X..) $y=9 E-05 x^{3}-0,0176 x^{2}+$ $0,6420 x+78 R^{2}=0,9998^{* *}$

(..o..) $y=0,0001 x^{3}-0,0218 x^{2}+$ $0,8083 x+73 R^{2}=0,9998^{* *}$

$(-\bullet) y=3 E-05 x^{3}-0,0056 x^{2}+$ $0,0764 x+46 \quad R^{2}=0,9999^{* *}$

(- -$) \mathrm{y}=5 \mathrm{E}-05 \mathrm{x}^{3}-0,0102 \mathrm{x}^{2}+$ $0,3486 x+38 R^{2}=0,9999^{\star *}$

(.). $Y=7 E-05 x^{3}-0,0142 x^{2}+$ $0,5896 x+54 R^{2}=1,0000^{* *}$ (..0.) $y=2 E-05 x^{3}-0,0050 x^{2}+$ $0,0868 x+65 R^{2}=0,9999^{* *}$

(.. $X$..) $y=3 E-05 x^{3}-0,0056 x^{2}+$ $0,0764 x+59 R^{2}=0,9998^{* *}$ $(-o-) y=0,0001 x^{3}-0,0019 x^{2}+$ $0,0699 x+66 R^{2}=1,0000^{* *}$

$(-\bullet) y=1 E-05 x^{3}-0,0025 x^{2}+$ $0,2159 x+36,90 R^{2}=0,9999 * *$

(-D -) $y=2 E-06 x^{3}-0,0003 x^{2}+$ $0,0944 x+46,50 R^{2}=0,9999^{* *}$

$(-) \mathrm{y}=2 \mathrm{E}-05 \mathrm{x}^{3}-0,0031 \mathrm{x}^{2}+$ $0,2461 x+51,50 R^{2}=1,0000^{* *}$ (..0.) $y=3 E-05 x^{3}-0,005 x^{2}+$ $0,2714 x+32,10 R^{2}=0,9998^{\star *}$ (.. $X ..) y=2 E-06 x^{3}+9 E-05 x^{2}+$ $00049 x+35,50 R^{2}=0,9999^{* *}$ (..o..) $y=4 E-05 x^{3}-0,0056 x^{2}+$ $0,2524 x+40,20 R^{2}=0,9999^{* *}$ tilada e mantidas a $25^{\circ} \mathrm{C}$, por 24 horas (Rodo et al., 1998).

Os dados foram submetidos à análise de variância. As médias foram apresentadas por meio de regressão polinomial. A seleção da função de melhor ajuste obedeceu ao maior coeficiente de determinação. Os dados referentes às avaliações de teor de água não foram analisados estatisticamente, considerando-se apenas as diferenças numéricas.

\section{RESULTADOS E DISCUSSÃO}

Após a exposição ao calor seco por 48 horas, houve redução da porcentagem de fungos, principalmente, de Aspergillus spp., nas sementes de tomate das cultivares Quadrado Mix e Topmec, que foram tratadas a 70; 72,5 e $75^{\circ} \mathrm{C}$ e, nas da cultivar Calmec VFN, que foram expostas a 72,5 e $75^{\circ} \mathrm{C}$ (Figura 1). Além disso, por ocasião desta avaliação, também foi constatado que houve redução acentuada da porcentagem de bactérias nas sementes da cultivar Topmec, que foram expostas a $70^{\circ} \mathrm{C}$ e nas da cultivar Calmec VFN, tratadas a $70 ; 72,5$ e $75^{\circ} \mathrm{C}$, cujos sintomas nas plântulas podem ter sido causados, principalmente, por Erwinia spp. Silva et al. (2002), também empregando o método de crescimento em gerbox, relataram que este apresenta dificuldades associadas à interpretação dos resultados e que proporciona baixa sensibilidade na detecção de bactérias, devido ao pequeno tamanho da amostra.

Também pode-se constatar na Figura 1 , que houve eliminação apenas de Aspergillus spp. nas sementes da cultivar Topmec, que foram tratadas a 70 ; 72,5 e $75^{\circ} \mathrm{C}$, por 120 horas e, nas da cultivar Quadrado Mix, que foram expostas a $75^{\circ} \mathrm{C}$, por 120 horas. No entanto, em sementes de tomate, Muniz (2001) observaram que o tratamento via calor seco, por 12 e 15 dias eliminou Alternaria Alternata, Fusarium spp. e Cladosporium flavum. Também, Silva et al. (2002) constataram controle de Xanthomonas campestris pv. vesicatoria, assim como de fungos dos gêneros Aspergillus e Rhizopus, após 96 horas de exposição ao calor. O tratamento térmico não foi suficiente para eliminar todos os microorganismos em sementes de tomate e que a eficiência deste tipo de tratamento depende além do patógeno, da cultivar da mesma espécie, como também relatado por Machado (2000).

Em relação à qualidade fisiológica das sementes de tomate das cultivares Quadrado Mix, Topmec e Calmec VFN, pode-se verificar que não houve diferentes comportamentos destas cultivares quanto à sensibilidade ao tratamen- 
Tabela 1. Teor de água das sementes de tomate das cultivares Quadrado Mix, Topmec e Calmec VFN, obtidos antes do tratamento térmico via calor seco e após o condicionamento osmótico. Rio de Janeiro, UFRRJ, 2001.

\begin{tabular}{|c|c|c|c|c|c|c|c|c|c|}
\hline \multirow{2}{*}{ Tratamentos } & \multicolumn{3}{|c|}{ Quadrado Mix } & \multicolumn{3}{|c|}{ Topmec } & \multicolumn{3}{|c|}{ Calmec VFN } \\
\hline & 70 & 72 & 75 & 70 & 72,5 & 75 & 70 & 72,5 & 75 \\
\hline \multirow[t]{2}{*}{$\begin{array}{l}\text { Periodos de } \\
\text { exposiçāo (h) }\end{array}$} & & & & & $\%$ & & & & \\
\hline & \multicolumn{9}{|c|}{ Antes do tratamento térmico } \\
\hline 0 & 10,47 & 10,61 & 10,91 & 10,67 & 10,61 & 10,18 & 10,09 & 10,55 & 10,30 \\
\hline 48 & 2,27 & 2,05 & 1,98 & 2,23 & 1,67 & 1,38 & 2,20 & 1,26 & 1,14 \\
\hline 96 & 2,03 & 1,66 & 1,36 & 2,05 & 1,28 & 1,10 & 1,94 & 1,06 & 1,05 \\
\hline \multirow[t]{2}{*}{120} & 1,45 & 1,38 & 1,18 & 1,73 & 1,18 & 1,00 & 1,92 & 1,00 & 1,00 \\
\hline & \multicolumn{9}{|c|}{ Após o condicionamento osmótico } \\
\hline 0 & 14,06 & 14,42 & 14,99 & 14,71 & 15,99 & 14,95 & 14,69 & 15,03 & 15,89 \\
\hline 48 & 13,28 & 13,36 & 15,26 & 14,07 & 14,14 & 14,18 & 14,11 & 14,27 & 14,42 \\
\hline 96 & 13,65 & 13,73 & 14,56 & 14,68 & 14,78 & 14,63 & 14,16 & 14,34 & 14,77 \\
\hline 120 & 13,68 & 13,83 & 15,48 & 14,66 & 15,15 & 14,25 & 14,62 & 14,69 & 14,99 \\
\hline
\end{tabular}

to térmico (Figura 2). Este resultados podem estar relacionados aos semelhantes teores iniciais de água das sementes destas cultivares (Tabela 1). Para Machado (2000), os efeitos da termoterapia são mais danosos, à medida que as sementes apresentam teores de água maiores, sendo que sementes mais secas são mais tolerantes às temperaturas mais altas do que sementes mais úmidas.

As sementes de tomate das três cultivares mantiveram a germinação após a exposição a $70 ; 72,5$ e $75^{\circ} \mathrm{C}$, por 48 horas (Figura 2), embora a termoterapia tenha promovido a redução de microorganismos presentes nas sementes após a exposição ao calor, por este período (Figura 1). Além disso, após a exposição a 70; 72 e $75^{\circ} \mathrm{C}$, por 96 horas, houve redução acentuada da germinação das sementes das três cultivares, sendo estes valores semelhantes aos constatados após a exposição ao calor, por 120 horas. No entanto, estes resultados foram discordantes aos encontrados por Muniz (2001) e Silva et al. (2002), pois para estes autores, os maiores períodos de exposição da semente de tomate ao calor não causaram danos à germinação das mesmas.

Quando as sementes das três cultivares foram submetidas a 70; 72,5 e $75^{\circ} \mathrm{C}$ por 48 horas, estas apresentaram queda do vigor avaliado pela maior lixiviação de exsudados e pela redução na porcentagem de plântulas normais na primeira contagem (Figura 2). Silva et al. (2002) também constataram redução do vigor avaliado pela primeira conta- gem, das sementes das amostras tratadas, sendo que esta queda foi compensada pelo melhor desenvolvimento das plântulas devido à ausência de patógenos, tanto de Erwinia spp. como de Aspergillus spp.

Neste trabalho, estes resultados provavelmente estão relacionados ao teor de água das sementes após o tratamento térmico, que foi reduzindo até atingir valores entre 1,75 e $1,0 \%$, por ocasião da avaliação realizada após 120 horas de exposição ao calor (Tabela 1). Em sementes de tomate, Silva et al. (2002) constataram que o teor de água pode ter prejudicado não só a velocidade de germinação, como o desenvolvimento de outros patógenos de sementes, como Aspergillus spp., Rhizopus spp. e Erwinia spp. Para Hobbs e Obendorf (1972), baixos teores de água das sementes provocam danos por embebição, levando ao menor desempenho, uma vez que a hidratação ocorre rapidamente, causando danos às membranas em reorganização. No entanto, resultados discordantes foram obtidos por Nakamura (1982) que constataram que o teor de água a $1 \%$, após o tratamento térmico a $70^{\circ} \mathrm{C}$ por 20 dias, não causou danos à qualidade fisiológica das sementes.

Quando foi realizado o condicionamento osmótico das sementes de tomate das cultivares Quadrado Mix, Topmec e Calmec VFN, previamente tratadas via calor seco, pode-se constatar que não houve diferentes respostas das cultivares (Figura 2). Para Mauromicale e Cavallaro (1995), o condicionamento favoreceu a germinação de sementes de tomate, dependendo da cultivar avaliada. De acordo com Penãloza e Eira (1993), as sementes dos lotes de baixa qualidade fisiológica precisam de maior período para a promoção da reorganização de suas membranas, visando tornarem propensas à germinação.

Houve aumento da germinação das sementes condicionadas das três cultivares, que foram previamente tratadas a 70; 72,5 e $75^{\circ} \mathrm{C}$, por zero, $48 ; 96$ e 120 horas, em relação às não condicionadas, sendo este aumento mais acentuado quando as sementes foram expostas por 48 horas (Figura 2). Além disso, pelos testes de condutividade elétrica e primeira contagem, foi evidenciado aumento do vigor das sementes condicionadas das três cultivares, que foram previamente expostas ao calor seco, em relação às não condicionadas, à semelhança do observado quanto à germinação (Figura 2). No presente trabalho, esses resultados podem estar relacionados com o grau de suscetibilidade das sementes de tomate à injúria provocada pela rápida embebição das sementes, que foram expostas ao calor seco por 96 e 120 horas, devido ao menor teor de água destas sementes (Tabela 1), como relatado por Vertucci (1989), que a eficiência de reorganização dos constituintes celulares depende da velocidade de hidratação, ou seja, dentre outros, do teor de água das sementes.

Pelos resultados obtidos, pode-se concluir que, dependendo da cultivar avaliada, o tratamento térmico não foi 
eficiente em eliminar os patógenos associados às sementes de tomate. Não houve diferença de comportamento das cultivares quanto a sensibilidade de exposição ao tratamento térmico via calor seco e ao condicionamento osmótico. Períodos prolongados de exposição ao calor seco causaram danos à qualidade fisiológica das sementes de tomate, que não foram recuperados com o subseqüente condicionamento osmótico.

\section{LITERATURA CITADA}

ALVARADO, A.D.; BRADFORD, K.L.; HEWITT, J.D. Osmotic priming of tomato seeds: effects on germination, field emergence, seedling growth and fruit yield. Journal of the American Society for Horticulture Science, v.112, n.3, p. 427 432, 1987.

BRASIL. Ministério da Agricultura e Reforma Agrária. Regras para análise de sementes. Brasília: SNDA/DNDV/CLAV, 1992. 365 p. CAVALLARO, V.; MAUROMICALE, G.; VINCENZO, G. Effects of seed osmoconditioning on emergence characteristics of the tomato (Lycopersicon esculentum Mill.). Acta Horticulturae, v.362, n.1, p.213-220, 1994.

FRETT, J.J.; PILL, W.G.; MORNEAU, D.C.A. A comparison of priming agents for tomato and asparagus seeds. HortScince, v.26, n.9, p.11581159, 1991.

HARDEGREE, S.P.; EMMERICH, W.E. Seed germination response to polyethylene glycol solution depth. Seed Science and Techonology, v.22, n.1, p.1-7, 1994.
HOBBS, P.R.; OBENDORF, R.L. Interactions of initial seed moisture and imbibitional temperature on germination and productivity of soybean. Crop Science, v.13, n.1, p.664-667, 1972.

MACHADO, J.C. Tratamento de sementes no controle de doenças. Lavras: LAPS/UFLA/ FAEPE, 2000. 138 p.

MARTINS, L.; SILVA, W.R.; ALMEIDA, R.R. Sanidade em sementes de Brachiaria brizantha (Hochst.ex A. Rich) Stapf submetidas a tratamentos térmicos e químicos. Revista Brasileira de Sementes, v.23, n.2, p.117-120, 2001.

MAUROMICALE, G.; CAVALLARO, V. Effects of seed osmopriming on germination of tomato at different water potential. Seed Science and Tecnhology, v.23, n.2, p.393-403, 1995.

MENTEN, J.O.M. Prejuízos causados por patógenos associados às sementes. In: MENTEN, J.O.M. (Ed.). Patógenos em sementes: detecção, danos e controle químico. São Paulo: CibaAgro, 1995. p.115-136.

MICHEL, B.E.; KAUFMANN, M.,R. The osmotic potential of polyethylene glycol 6000 . Plant Physiology, v.51, n.8, p.914-916, 1973.

MUHYADDIN, T.; WIEBE, H.J. Effects of seed treatments with polyethylene glycol on emergence of vegetable crops. Seed Science and Technology, v.17, n.1, p.49-56, 1989.

MUNIZ, M.F.B. Controle de microrganismos associados às sementes de tomate através do uso do calor seco. Revista Brasileira de Sementes, v.23, n.1, p.276-280, 2001.

NAKAMURA, H. Effects of dry heat treatment for seed desinfection on germination on vegetables. Journal Agricultural Research, v.15, n.4, p.243-247, 1982.

PENÃLOZA, A.P.S.; EIRA, M.T.S. Hydrationdehydration treatments on tomate seeds (Lycopersicum esculentum Mill). Seed Science and Techonology, v.21, n.3, p.309-316, 1993.
PERLEBERG, C.S.; SPERANDIO, C.A. Influência da termoterapia na qualidade sanitária e fisiológica de sementes de arroz. Revista Brasileira de Sementes, v.20, n.2, p.311-316, 1998.

PILL, W.G.; FRETT, J.J.; MORNEAU, D.C. Germination and seedling emergence of primed tomato and asparagus seeds under adverse conditions. HortScience, v.26, n.9, p.1160-1162, 1991.

RODO, A B.; TILLMANN, M.A.A.; VILLELA, F.A. Testes de vigor na avaliação da qualidade fisiológica de sementes de tomate. Revista Brasileira de Sementes, v.20, n.1, p.23-28, 1998.

ROSSETTO, C.A.V.; LIMA, T.M.; NAKAGAWA, J. Avaliação da aplicação de fungicida e do condicionamento osmótico durante o armazenamento de sementes de tomate. Revista Brasileira de Sementes, v.23, n.2, p.229-235, 2001.

ROSSETTO, C.A.V.; LIMA, T.M.; NAKAGAWA, J. Qualidade fisiológica e potencial de armazenamento de sementes de tomate submetidas ao condicionamento osmótico. Horticultura Brasileira, Brasília, v.20, n.4, p.630634, 2002.

SILVA, A.M.S.; CARMO, M.G.F.; OLIVARES, F.L.; PEREIRA, A.J. Termoterapia via calor seco no tratamento de sementes de tomate: eficiência na erradicação de Xanthomonas campestri pv. vesicatoria e efeitos sobre a semente. Fitopatologia Brasileira, Brasília, v.27, n.6, p.586593, 2002.

TRIGO, M.F.O.O.; PIEROBOM, C.R.; NEDEL, J.L.; TRIGO, L.F.N. Efeito da pré-secagem sobre o desempenho de sementes de cenoura na termoterapia. Revista Brasileira de Sementes, v.20, n.1, p.43-47, 1998.

VERTUCCI, C.W. The kinectis of seed imbibition. In: CROP SCIENCE SOCIETY OF AMERICA. Seed Moisture. Madison: CSSA, 1989. p.93-115 (CSSA. Special Publication, 14). 